\title{
Queratocisto odontogênico: índice de recorrência em função do tipo de tratamento
}

Dental keratocyst: recurrence rate due to the type of treatment

Queratoquiste odontogénico: indicación de recurrencia por tipo de tratamiento

Mateus Henrique de OLIVEIRA

Cirurgião-dentista. Faculdade de Odontologia da Universidade Federal de Mato Grosso do Su

Maisa de Oliveira AGUILLERA

Residência em Cirurgia e Traumatologia Bucomaxilofacial da Faculdade de Odontologia e Hospital Universitário "Maria Aparecida Pedrossian" da Universidade Federal de Mato Grosso do Su https://orcid.org/0000-0003-0055-6984

Muryllo Eduardo Sales dos SANTOS

Residência em Cirurgia e Traumatologia Bucomaxilofacial da Faculdade de Odontologia e Hospital Universitário "Maria Aparecida Pedrossian" da Universidade Federal de Mato Grosso do Sul https://orcid.org/0000-0001-8517-4154

Francielly Thomas FIGUEIREDO

Residência em Cirurgia e Traumatologia Bucomaxilofacial da Faculdade de Odontologia e Hospital Universitário "Maria Aparecida Pedrossian" da Universidade Federal de Mato Grosso do Su https://orcid.org/0000-0003-0196-1172

Yuri NEJAIM

Preceptor da Residência em Cirurgia e Traumatologia Bucomaxilofacial da Faculdade de Odontologia e Hospital Universitário "Maria Aparecida Pedrossian" da Universidade Federal de Mato Grosso do Sul https://orcid.org/0000-0002-0442-2601 Daniella Moraes ANTUNES

Preceptora da Residência em Cirurgia e Traumatologia Bucomaxilofacial da Faculdade de Odontologia e Hospital Universitário "Maria Aparecida Pedrossian" da Universidade Federal de Mato Grosso do Sul https://orcid.org/0000-0002-7557-5461 Julio Cesar Leite da SILVA

Preceptor da Residência em Cirurgia e Traumatologia Bucomaxilofacial da Faculdade de Odontologia e Hospital Universitário "Maria Aparecida Pedrossian" da Universidade Federal de Mato Grosso do Su https://orcid.org/0000-0002-9835-7432 Rosana Leite de MELO

Preceptora da Residência em Cirurgia e Traumatologia Bucomaxilofacial da Faculdade de Odontologia e Hospital Universitário "Maria Aparecida Pedrossian" da Universidade Federal de Mato Grosso do Sul Ellen Cristina GAETTI JARDIM

Preceptora da Residência em Cirurgia e Traumatologia Bucomaxilofacial da Faculdade de Odontologia e Hospital Universitário "Maria Aparecida Pedrossian" da Universidade Federal de Mato Grosso do Sul https://orcid.org/0000-0003-2471-465X

\section{Resumo}

O queratocisto odontogênico é um cisto de desenvolvimento com alto poder de recorrência que acomete principalmente a região posterior de mandibula sendo muitas vezes assintomático e diagnosticado somente a partir de um achado imaginológico. Seu tratamento pode variar desde a descompressão cística seguida de enucleação com auxílio de técnicas complementares como a osteotomia periférica ate uma intervenção mais agressiva como a ressecção em bloco da região envolvida. Desde modo, em virtude do índice de recidiva e a gama de tratamentos existentes este trabalho tem como objetivo realizar uma revisão de literatura para analisar uma possível relação entre a porcentagem de recorrência e a escolha do tipo de tratamento. Para tal, foi realizada uma pesquisa bibliográfica tendo como base de dados PUBMED, SCIELO e MEDLINE. Foram incluídos os artigos que tenham o tema queratocisto odontogênico ou tumor odontogênico queratocístico e suas abordagens cirúrgicas de tratamento. de acordo com a classificação da Organização Mundial da Saúde. Os estudos concluíram que pode haver uma correlação entre o tratamento mais agressivo e o menor índice de recorrência da lesão.

Descritores: Cistos Odontogênicos; Terapêutica; Recidiva.

\section{Abstract}

Odontogenic keratocyst is a developmental cyst with a high power of recurrence that mainly affects the posterior region of the mandible and is often asymptomatic and diagnosed only on the basis of an imaging finding. Its treatment can vary from cystic decompression followed by enucleation with the aid of complementary techniques such as peripheral osteotomy to a more aggressive intervention such as en bloc resection of the region involved. Therefore, due to the recurrence rate and the range of existing treatments, this study aims to conduct a literature review to analyze a possible relationship between the percentage of recurrence and the choice of type of treatment. To this end, a bibliographic search was performed using the PUBMED, SCIELO and MEDLINE database. Articles with the theme odontogenic keratocyst or keratocystic odontogenic tumor and their surgical treatment approaches were included. according to the classification of the World Health Organization. The studies concluded that there may be a correlation between the most aggressive treatment and the lowest injury recurrence rate.

Descriptors: Odontogenic Cysts; Therapeutics; Recurrence.

\section{Resumen}

El queratoquiste odontogénico es un quiste del desarrollo con un alto poder de recidiva que afecta principalmente a la región posterior de la mandíbula, a menudo es asintomático $\mathrm{Y}$ se diagnostica solo a partir de un hallazgo de imagen. Su tratamiento puede variar desde la descompresión quística seguida de enucleación con la ayuda de técnicas complementarias como la osteotomía periférica hasta una intervención más agresiva como la resección en bloque de la región afectada. Por tanto, debido a la tasa de recurrencia $Y$ la variedad de tratamientos existentes, este estudio tiene como objetivo realizar una revisión de la literatura para analizar una posible relación entre el porcentaje de recurrencia Y la elección del tipo de tratamiento. Para ello, se realizó una búsqueda bibliográfica utilizando la base de datos PUBMED, SCIELO Y MEDLINE. Se incluyeron artículos que incluían el tema queratoquiste odontogénico o tumor queratoquístico odontogénico $Y$ sus enfoques de tratamiento quirúrgico. Según la clasificación de la Organización Mundial de la Salud, los estudios concluyeron que puede haber una correlación entre el tratamiento más agresivo Y la tasa más baja de recurrencia de lesiones.

Descriptores: Quistes Odontogénicos; Terapéutica; Recurrencia. INTRODUÇÃO

O queratocisto odontogênico é um cisto
de desenvolvimento caracterizado por

revestimento de epitélio queratinizado e comportamento agressivo. Representa aproximadamente $11 \%$ de todos os cistos 
odontogênicos da região maxilofacial e seu tratamento se mostra desafiador devido ao seu grande potencial de crescimento e altas taxas de recidiva ${ }^{1-3}$.

A terminologia "Queratocisto" surgiu na década de 1950 para representar qualquer tipo de cisto que possuísse queratinização. Alguns anos depois, em 1971, a Organização Mundial da Saúde (OMS) tornou Queratocisto a terminologia oficial para descrever um tipo específico de cisto odontogênico ${ }^{4}$. Existia então dois subtipos histopatológicos de Queratocisto Odontogênico: ortoqueratinizado e paraqueratinizado $^{5}$ sendo que 0 paraqueratinizado tinha associação com a Síndrome do Carcinoma Nevoide Basocelular (SCNBC) e apresentava maiores taxas de recidivas.

Por este motivo, em 2005 a OMS deixou de lado o termo Queratocisto Odontogênico ${ }^{6}$. O subtipo paraqueratinizado passou então a se chamar "tumor odontogênico queratocístico" (TOQ), enquanto o subtipo ortoqueratinizado ganhou a terminologia de "cisto odontogênico ortoqueratinizado", continuando como cisto de desenvolvimento odontogênico. Há dois anos, em 2017, o TOQ voltou para a classificação da OMS de "cistos odontogênicos de desenvolvimento" mantendo os critérios histopatológicos determinados em 2005 e permanecendo distinto do cisto odontogênico ortoqueratinizado ${ }^{2}$.

O tratamento do queratocisto odontogênico ainda é controverso. A experiência de cada profissional ainda é a maneira adotada para a eleição do tratamento adequado. ${ }^{7}$ As técnicas cirúrgicas variam desde tratamento conservadores como a enucleação, até tratamentos mais agressivos com ressecções parciais ou totais ${ }^{7-9}$.

Neste sentido, o estudo se propôs a revisar as diferentes formas de tratamento do queratocisto com objetivo de avaliar as taxas de recorrências. Além disso, teve como proposito comparar os resultados entre as formas de tratamento e taxas de recidiva.

REVISÃO DA LITERATURA

Os cistos e tumores odontogênicos são frequentes na patologia oral e maxilofacial. Os cistos são os mais comuns encontrados na pratica odontológica e vale a cada profissional o diagnóstico correto para manejo do tratamento $^{10}$.

Cistos odontogênicos derivam, em grande parte, do epitélio odontogênicos em sua maioria e são subclassificados de acordo com sua origem como cistos de desenvolvimento e cistos inflamatórios. O queratocisto é um cisto de desenvolvimento que surge dos restos celulares da lâmina dentaria. Seu crescimento está relacionado a fatores genéticos inerentes do seu próprio epitélio ou de atividade enzimática na parede fibrosa do cisto e não por pressão osmótica ${ }^{3}$.

O queratocisto foi reconhecido como uma lesão neoplásica na classificação da OMS em 2005 nomeado como tumor odontogênico queratocístico ${ }^{6}$, porém em uma última classificação pela OMS (2017), os queratocistos voltam a ser classificados como lesão cística, e - cisto odontogênico ortoqueratinizado é identificado como uma variante do queratocisto ${ }^{2}$.

Apesar das características recidivantes e crescimento agressivo do queratocisto após o tratamento, a nomenclatura foi reclassificada pela Organização Mundial da Saúde em $2017^{11}$. O termo anteriormente descrito como Tumor Odontogênico Queratocístico passou a ser considerado como Queratocisto Odontogênico. Essas mudanças buscam estabelecer um padrão de linguagem no meio científico para que o diagnóstico de patologias como os tumores e cistos de cabeça e pescoço não sejam confundidos e tratados erroneamente ${ }^{12}$.

$O$ diagnóstico diferencial com 0 queratocisto pode ser o ameloblastoma, cisto dentígero, cisto radicular, granuloma central de células gigantes, cisto ósseo traumático, cisto de Gorlin, cisto residual e mixoma odontogênico ${ }^{13}$.

O queratocisto odontogênico tem prevalência em pacientes com idade acima dos 50 anos em $37,7 \%$ dos casos e de $28,56 \%$, abaixo dos 20 anos. Apresenta predileção ao sexo masculino e etnia branca, $67,8 \%$, dos casos $^{14}$. Em $40 \%$ dos casos há associação com um dente não erupcionado ${ }^{15}$.

O seu tratamento ainda é controverso. A experiência de cada profissional ainda é a maneira adotada para a eleição do tratamento adequado ${ }^{7}$. As técnicas cirúrgicas variam desde tratamento conservadores como a enucleação, até tratamentos mais agressivos com ressecções parciais ou totais ${ }^{7-9}$.

A enucleação com osteotomia periférica tem se mostrado eficaz no tratamento do queratocisto e apresenta menor morbidade ${ }^{16}$. Para a diminuição das recorrências, existem tratamentos coadjuvantes como a utilização de solução de Carnoy e a crioterapia, os quais penetram no osso adjacente causando uma destruição química das células tumorais remanescentes em até $1.5 \mathrm{~mm}^{17,18}$.

A aplicação da solução de Carnoy como terapia adjuvante ajuda a diminuir 
significativamente a recorrência do cisto ${ }^{18}$.

Em grandes lesões apontam a técnica da descompressão com irrigações periódicas com o objetivo de diminuir a pressão intralesional permitindo a reparação óssea adjacente e consequente diminuição da lesão ${ }^{14}$.

Em relação á recorrências relacionadas ao tipo de tratamento, diversos estudos existem na literatura. As técnicas enucleação, curetagem e marsupialização estão mais relacionadas a maior taxa de recorrência devido aos tecidos remanescentes da lesão ${ }^{19}$, já a ressecção radical é o tratamento que apresenta a menor taxa de recorrência ${ }^{9}$.

Quando da natureza agressiva, na maioria das vezes, o tratamento igualmente agressivo se faz necessário ${ }^{20}$. A recorrência da lesão parece estar mais relacionada à natureza do queratocisto do que a técnica utilizada para seu tratamento, devido ao seu potencial de formar cistos satélites a distância, a característica friável de sua capsula e formação de septos e bordas irregulares, dificultando a sua total remoção pelas técnicas mais conservadoras $^{21}$, desta forma, 0 tipo de tratamento não determinará por si só o risco de recidiva. Uma análise comparativa entre diferentes modalidades de tratamento e recorrência é apresentada na tabela 1.

Tabela 1. Análise comparativa de diferentes modalidades de tratamento e índice de recorrência para o tratamento do queratocisto odontogênico

\begin{tabular}{|c|c|c|c|c|c|c|c|c|}
\hline Artigos & En & Ds+En & Mr+En & $\mathbf{E n}+\mathbf{S C}$ & $\mathbf{E n}+\mathbf{C r}$ & En+OP & $\mathrm{En}+\mathbf{O P}+\mathrm{SC}$ & $\mathbf{R B}$ \\
\hline $\begin{array}{c}\text { Balmick et } \\
\text { al. }^{21}\end{array}$ & $71,42 \%$ & - & - & $42,85 \%$ & - & - & - & $0 \%$ \\
\hline Berge et al. ${ }^{22}$ & $6 \%$ & - & $9 \%$ & - & - & - & - & - \\
\hline $\begin{array}{l}\text { Chrcanovic } \\
\text { et al.23 }\end{array}$ & $22.5 \%$ & $18.6 \%$ & $28,7 \%$ & $5 \cdot 3 \%$ & $20.9 \%$ & $18.6 \%$ & - & $2.2 \%$ \\
\hline $\begin{array}{c}\text { Ribeiro- } \\
\text { Júnior et al.17 }\end{array}$ & $14,8 \%$ & - & $15.4 \%$ & $14,3 \%$ & - & $10 \%$ & - & - \\
\hline $\begin{array}{l}\text { Alchalabi } \\
\text { et al. }{ }^{24}\end{array}$ & - & - & - & - & - & - & $0 \%$ & - \\
\hline $\begin{array}{l}\text { Al-moraissi } \\
\text { et al. }{ }^{9}\end{array}$ & $23,1 \%$ & - & $32,3 \%$ & 11,5\% & $14,5 \%$ & $17,4 \%$ & - & $8,4 \%$ \\
\hline $\begin{array}{c}\text { de Souza } \\
\text { Cruz et al.25 }\end{array}$ & - & - & - & - & $20 \%$ & - & - & - \\
\hline $\begin{array}{c}\text { Spivakovsky } \\
\text { et al. }{ }^{26}\end{array}$ & $20,8 \%$ & $11.9 \%$ & $18,2 \%$ & - & - & - & - & - \\
\hline $\begin{array}{l}\text { de Castro } \\
\text { et al. } 27\end{array}$ & $20.8 \%$ & $11,9 \%$ & - & - & - & - & - & - \\
\hline $\begin{array}{c}\text { Slusarenko } \\
\text { da Silva } \\
\text { et al. }{ }^{28}\end{array}$ & $28.8 \%$ & - & $19.4 \%$ & - & - & - & - & - \\
\hline Fidele et al 7 & $95 \%$ & - & - & - & - & - & - & - \\
\hline $\begin{array}{l}\text { Tabrizi } \\
\text { et al.29 }\end{array}$ & - & $14,2 \%$ & $27,12 \%$ & - & - & - & - & - \\
\hline \multicolumn{9}{|c|}{$\begin{array}{l}\text { Legenda: En = Enucleação; } \\
\text { Ds+En = Descompressão + Enucleação; } \\
M r+E n=\text { Marsupialização + Enucleação } \\
E n+S C=\text { Enucleação + Solução de Carnoy } \\
\text { En + Cr = Enucleação + Crioterapia } \\
\text { En + OP = Enucleação + Osteotomia Periférica } \\
\text { En + OP + SC = Enucleação + Osteotomia Periférica + Solução de Carnoy } \\
\text { RB = Ressecção em bloco }\end{array}$} \\
\hline \multicolumn{9}{|c|}{ DISCUSSÃO } \\
\hline
\end{tabular}

O queratocisto odontogênico desde a primeira classificação da OMS vem sofrendo modificações na sua nomenclatura. Até 1956 o mesmo se enquadrava na categoria de cisto primordial $^{13}$ em contrapartida, em 2005 como tumor odontogênico queratocístico (TOQ) e atualmente na categoria de cisto odontogênico; os tipos de tratamento empregados nestes casos variaram de cirúrgicos conservadores a radicais com ressecção de grande margem óssea ${ }^{2,9}$.

Em grandes lesões, a descompressão com irrigações periódicas com o objetivo de diminuir a pressão intralesional permitindo a reparação óssea adjacente e consequente diminuição da lesão ${ }^{14}$.

Em um estudo realizado por Kahraman et al. ${ }^{1}$, foram analisados 204 casos de queratocistos em um período de 7 anos na Turquia. Os queratocistos foram agrupados de acordo com a frequência de cistos satélites e ilhas escamosas dentro da parede do cisto. O estudo revelou que a maioria dos queratocistos se apresenta no tipo unicístico sem cistos satélites associados compreendendo $63 \%$ dos casos, $27 \%$ apresentaram número variável de cistos satélites e ilhas escamosas em seu interior, e apenas $6 \%$ numerosos cistos satélites e ilhas escamosas.

Estes resultados são mencionados também por Tabrizi et al. ${ }^{29}$, em 2014, que enfatizam o alto poder de recorrência dos QO multiloculares. Além disso, o artigo mostra que para cada aumento de $1 \mathrm{~cm}$ no tamanho da lesão a taxa de reincidência aumenta 1,67 vezes.

Chrcanovic et al. ${ }^{23}$ em 2017 realizaram um estudo onde incluíram 6427 casos de queratocistos com recorrências em 1464 casos, representando um índice de recorrência total entre todas as modalidades de tratamento em $21,1 \%$. Em relação ao manejo terapêutico, o mais alto índice de recorrência foi representado pela marsupialização com $28.7 \%$ e enucleação com $22.5 \%$. O menor índice de recorrência teve como tratamento a enucleação e uso de solução de Carnoy (5.3\%), ressecção marginal (2.2\%). A descompressão seguida da enucleação representou $11.3 \%$ de recidiva em comparação com somente a descompressão.

De Castro et a. ${ }^{27}$ realizaram um estudo comparativo entre 3 tipos de tratamentos conservadores para pacientes que apresentavam QO: descompressão seguida de enucleação, marsupialização mais enucleação e enucleação simples. As taxas de recorrência para a lesão nos pacientes foram de 11,9\%; $17,8 \%$; e $20,8 \%$ respectivamente. Notou-se através dos resultados os benefícios de se realizar um procedimento cirúrgico de descompressão ou marsupialização previamente à enucleação da lesão, pois diminui o índice de recorrência.

Neste mesmo sentido, Tabrizi et al. $^{29}$, realizaram uma revisão sistemática avaliando a 
eficácia da utilização dos tratamentos conservadores de descompressão e marsupialização. Foram avaliados 341 pacientes com um tempo medico de acompanhamento de 58.30+-32,80 meses. 182 pacientes foram submetidos a marsupialização, 118 foram tratados apenas com a marsupialização e 64 tivera a enucleação ou cistectomia como adjuvante, outro grupo de pacientes com 159 receberam o tratamento de descompressão, 74 sem outro tratamento adjuvante e 85 foram tratados com enucleação ou cistectomia. Dos tratamentos conservadores utilizados sem nenhuma intervenção extra, $27,12 \%$ dos pacientes que realizaram marsupialização tiveram recidiva, e somente $5.4 \%$ dos pacientes submetidos apenas a descompressão, apresentaram recidiva.

Em análise bibliográfica, observamos que o tratamento que mais se associa a recidivas é a enucleação sem tratamentos coadjuvantes com uma média de $33,7 \%$ dos casos, e estes quando associado a descompressão previa, apresentam melhor prognóstico, reduzindo a um índice de 18,5\%. Estudos relataram que após a marsupialização, a cápsula fibrosa torna-se mais espessa e menos friável, facilitando 0 procedimento cirúrgico de enucleação e reduzindo a taxa de recorrência ${ }^{30}$.

A utilização de técnicas complementares para o tratamento dos cistos e tumores odontogênicos como a aplicação da solução de Carnoy na cavidade da lesão, promove uma necrose química superficial em até $1,5 \mathrm{~mm}$ eliminando remanescentes teciduais do tumor reduzindo assim as taxas de recidiva ${ }^{17,31}$, eliminando as ilhas epiteliais e cistos satélites nos limites da lesão ${ }^{31}$.

Diaz-Belenguer et al. $^{32}$ realizaram uma revisão sistemática para análise de recorrência dos queratocistos após uso de solução de Carnoy. Foram analisados 13 trabalhos de série de casos onde mostrou uma taxa de recorrência após uso do agente químico após enucleação variando de $0 \%$ a $100 \%$, porém utilizando duas ou mais técnicas adjuvantes, reduz a faixa de recidiva de $0 \%$ a $7.9 \%$ concluindo então que 0 tratamento coadjuvante com a solução de Carnoy tem uma recomendação à critério do cirurgião sendo necessário realizar novos estudos para comprovação da eficácia.

Em contrapartida, os resultados obtidos por Gosau et al. ${ }^{18}$ demonstram uma redução para $14,3 \%$ na taxa de recidiva para os pacientes submetidos a aplicação da solução de Carnoy posteriormente à enucleação. Já, Alchalabi et al. ${ }^{24}$ realizaram um estudo retrospectivo de 29 pacientes com diagnóstico de queratocisto odontogênico tratados mediante enucleação seguido de osteotomia periférica e aplicação de solução de Carnoy como método padrão e em acompanhamento de 7 anos não houveram recidivas. Nesse mesmo ano de 2017 Moraissi et al., apresentou pacientes tratados a partir da enucleação mais ostectomia periférica que obtiveram uma taxa de recorrência de $17,4 \%$. Portanto, a ostectomia periférica como opção complementar do tratamento para o QO tem grande importância no combate à reincidência da lesão.

Outro método coadjuvante para o tratamento do queratocisto odontogênico encontrado na literatura é o uso da crioterapia. de Souza Cruz et al. ${ }^{25}$ realizaram um estudo com 10 pacientes submetidos a marsupialização seguido de enucleação e crioterapia, apresentando apenas 2 casos de recidiva da lesão. A crioterapia resulta no congelamento e descongelamento do tecido de interesse a uma temperatura em torno de $-20^{\circ} \mathrm{C}$ a $-40^{\circ} \mathrm{C}$ causando lesão no tecido atingido como descrito por Costa Jr et al. ${ }^{33}$ A recorrência observada no estudo pode ser relacionada a baixa resposta à marsupialização apresentada após 12 meses, corroborado por Cunha et al. ${ }^{34}$ em que referem que a presença de dentes associados a lesão, baixa resposta a descompressão e presença de células na camada basal provenientes das ilhas epiteliais na capsula fibrosa possa causar um novo brotamento, causando recidivas. A grande diferença na incidência de recidiva nos diferentes tratamentos evidencia ainda mais que a técnica empregada não está relacionada diretamente ao índice de recorrência.

Isto vem salientar a relevância do trabalho em confrontar o tratamento empregado com os índices de recorrência associados as mesmas uma vez que a literatura é vasta no tocante a recidiva elevada na presença do referido cisto.

Pela natureza agressiva do queratocisto, muitas vezes é necessária uma abordagem igualmente agressiva. Neste sentido, Berge et al. ${ }^{22}$ empregou como terapêutica em casos de TOQ com perfuração óssea e presença de delgada cápsula, a marsupialização associada a posterior enucleação da lesão encontrando 24\% de recidivas. Tal fato vem ao encontro do que foi mencionado por Goddard et $\mathrm{al}^{.8}$ que em casos de recidiva o tratamento deverá ser agressivo com a ressecção do bloco ósseo em presença desse tipo de tumor.

A literatura nos mostra que QO tratados com ressecção (hemimandibulectomia) o índice 
de sucesso com diminuição da reincidência da lesão foi maior, o que também pode ser comparado com o artigo de Fidele et al. ${ }^{7}$ que mencionam altos níveis de recorrência quando se lança mão de apenas o uso da enucleação como forma de tratamento o que nem sempre está atrelado a experiência do operador.

Warburton et al. ${ }^{34}$, também citam a eficácia do tratamento agressivo para O TOQ. Em sua pesquisa 12 pacientes foram submetidos à ressecção e nenhum deles apresentaram recorrência da lesão patológica. $\mathrm{Em}$ contrapartida, Al-moraissi et al. ${ }^{9}$ relata taxa de recidiva de $8.4 \%$ para os pacientes tratados através da ressecção.

Em contrapartida, de um total de 31 pacientes submetidos a mandibulectomia segmentar e posteriormente reconstruídos com retalhos vascularizados, Fidele et al. $^{7}$ relatam apenas 1 caso de recidiva quando do tratamento do QO fato este já demonstrado por Madras e Lapointe ${ }^{36}$ em que a taxa de sucesso alta no tratamento neste caso de um tumor odontogênico teve forte correlação com o tratamento mais radical através da ressecção em bloco. Este fato nos mostra que por menores que sejam as chances de recidiva da lesão nos pacientes tratados com ressecção, este tratamento agressivo não elimina a probabilidade de recorrência.

CONCLUSÃO

Em virtude do que foi exposto, percebese que a correlação do tratamento empregado e o índice de recidiva, não provém apenas da experiência do operador e sim da característica do queratocisto odontogênico. Observou-se que quando se associa mais técnicas adjuvantes a um tratamento conservador ou agressivo, a ocorrência de recidivas decresce.

REFERÊNCIAS

1. Kahraman D, Gunhan O, Celasun B. A series of 240 odontogenic keratocysts: Should we continue to use the terminology of 'keratocystic odontogenic tumour' for the solid variant of odontogenic keratocyst?. J Craniomaxillofac Surg. 2018;46(6):942-46.

2. Speight PM, Takata T. New tumour entities in the 4th edition of the World Health Organization Classification of Head and Neck tumours odontogenic and maxillofacial bone tumours. Vichows Arch. 2018, 472:331-39.

3. Neville BW, Allen CM, Damm DD, Chi AC. Patologia Oral e Maxilofacial. 4. ed. Rio de Janeiro: Elsevier; 2016.

4. Pindborg JJ, Kramer IRH, Torloni $\mathrm{H}$, World Health Organization. Tipos histológicos de tumores odontogénicos, quistes de los maxilares y lesiones afines. Ginebra: Organizacion Mundial de la Salud. 1972.

5. Forssell K, Kallioniemi H, Sainio P. Microcysts and epithelial islands in primoridal cysts. Proceedings of the Finnish Dental Society. Suomen Hammaslaakariseuran toimituksia. 1979;75(5-6):99-102.

6. Barnes L, Eveson JW, Reichart P, Sidransky D. Keratocystic odontogenic tumors in World Health Organization classification of tumours. Pathology and genetics of head and neck tumours. Lyon (France): IARC. 2005; p. 306-7.

7. Fidele NB, Yueyu Z, Zhao Y, Tianfu W, Liu J, Sun Y, Liu B. Recurrence of odontogenic keratocysts and possible prognostic factors: Review of 455 patients. Med Oral Patol Oral Cir Bucal. 2019;24 (4):e491-501.

8. Goddard AC, Brooks JH, Lewis RH, Lewis PS. Treatment for Recurrent Odontogenic Keratocysts. J Tenn Dent Assoc. 2016; 96(1):20-6.

9. Al-moraissi EA, Dahan AA, Alwadeai MS, Oginni FO, Al-jamali JM, Alkhutari AS et al. What surgical treatment has the lowest recurrence rate following the management of keratocystic odontogenic tumor? A large systematic review and meta-analysis. J Craniomaxillofac Surg. 2017;45:131-44.

10. Meningaud JP, Oprean N, Pitak-Arnnop P, Bertrand JC. Odontogenic cysts: a clinical study of 695 cases. J Oral Sci. 2006;48(2):59-62.

11. Chan JKC, EI-Naggar AK, Grandis JR, Takata T, Slootweg PJ. Who classification of head and neck tumours. World Health Organization.4. ed. 2017

12. Wright JM, Vered M. Update from the 4th edition of the world health organization classification of head and neck tumours: odontogenic and maxillofacial bone tumors. Head Neck Pathol. 2017;11(1):68-77.

13. Moura BS, Cavalcante MA, Hespanhol W. Tumor odontogênico ceratocístico. Rev Col Bras Cir. 2016;43(6):466-71.

14. Fonseca EV, Franzi SA, Marcucci M, Almeida RC. Fatores clínicos, histopatológicos e tratamento do tumor queratocisto odontogênico. Rev Bras Cir Cabeça Pescoço. 2010;39(1): 57-61.

15. Llauradó AV, Montero RA, Olmo IT, Enric JS, Roig AM, Lopez J. Opciones terapéuticas en quistes odontogénicos: revisión. Av Odontoestomatol. 2013;29(2):81-93.

16. Karaca C, Dere KA, Er N, Aktaş A, Tosun E, Köseoğlu $O$ et al.Recurrence rate of odontogenic keratocyst treated by enucleation and peripheral ostectomy: Retrospective case series with up to 12 years of follow-up. Med Oral Patol Oral Cir Bucal. 2018;23(4):e443-48.

17. Ribeiro Junior O, Borba AM, Alves CAF, Guimarães Junior J. Complicações da solução 
de Carnoy no tratamento de tumores odontogênicos. RGO. 2007;55(3):263-66.

18. Gosau M, Draenert FG, Müller S, Frerich B, Bürgers R, Reichert TE et al. Two modifications in the treatment of keratocystic odontogenic tumors (KCOT) and the use of carnoy's solution (CS)-a retrospective study lasting between 2 and 10 years. Clin Oral Investig. 2010;14:27-34.

19. Freitas DA, Veloso DA, Santos ALDA, Freitas VA. Maxillary odontogenic keratocyst: a clinical case report. RGO. 2015;63(4):484-88.

20. Kolokhytas A, Fernandes RP, Pazoki A, Ord RA. Odontogenic keratocyst: To decompress or not to decompress? A comparative study of decompression and enucleation versus resection/peripheral ostectomy. J Oral Maxillofac Surg. 2007;65(4):640-44.

21. Balmick S, Hespanhol W, Cavalcante MADA, Gandelmann IHA. Recidiva do tumor odontogênico ceratocístico: análise retrospectiva de 10 anos. Rev cir traumatol buco-maxilo-fac. 2011;11(1):85-91.

22. Berge $\mathrm{TI}$, Helland $\mathrm{SB}$, Sælen $A$, Øren $M$, Johannessen AC, Skartveit L, Grung B, Pattern of recurrence of non-syndromic keratocystic odontogenic tumours, Oral Surgery Oral Med Oral Pathol Oral Radiol. 2016;122(1):10-6.

23. Chrcanovic BR, Gomez RS. Recurrence probability for keratocystic odontogenic tumors: An analysis of 6427 cases. J CranioMaxillofac Surg. 2017;45(2):244-51.

24. Alchalabi NJ, Merza AM, Issa SA. Using Crnoy's Solution in Tratamento of Keratocystic Odontogenic Tumor. Ann Maxillofac Surg. 2017;7(1):51-6

25. de Souza Cruz EL, da Silva Tabosa AK, Falcão ASC, Tartari T, de Menezes LM, da Costa ET, Carneiro-Júnior JT. Use of refrigerant spray of a propane/butane/isobutane gas mixture in the management of keratocystic odontogenic tumors: a preliminary study. Oral Maxillofac Surg. 2017;21(1):21-6.

26. Spivakovsky, S. Surgical treatments for nonsyndromic odontogenic keratocysts. Evidence-based Dent. 2018;19(2):55-6.

27. de Castro MS, Caixeta CA, de Carli ML, Ribeiro Júnior NV, Miyazawa M, Pereira AAC, Sperandio FF, Hanemann JAC. Conservative surgical treatments for nonsyndromic odontogenic keratocysts: a systematic review and meta-analysis. Conservative surgical treatments for nonsyndromic odontogenic keratocysts: a systematic review and metaanalysis. Clin Oral Investig. 2018;22(5): 2089-101.

28. Slusarenko da Silva Y, Stoelinga PJW, Naclério-Homem MDG. Recurrence of nonsyndromic odontogenic keratocyst after marsupialization and delayed enucleation vs. enucleation alone: a systematic review and meta-analysis. Oral Maxillofac Surg. 2019; 23(1):1-11.

29. Tabrizi R, Hosseini Kordkheili MR, Jafarian M, Aghdashi F. Decompression or Marsupialization; Which Conservative Treatment is Associated with Low Recurrence Rate in Keratocystic Odontogenic Tumors? A Systematic Review. J Dent (Shiraz). 2019; 20(3):145-51.

30. Telles DC, Castro WH, Gomez RS, Souto GR, Mesquita RA. Morphometric evaluation of keratocystic odontogenic tumor before and after marsupialization. Braz Oral Res. 2013;27(6): 496-502.

31. Tolstunov L, Treasure T. Surgical treatment algorithm for odontogenic keratocyst: combined treatment of odontogenic keratocyst and mandibular defect with marsupialization, enucleation, iliac crest bone graft, and dental implants. J oral maxillofac surg. 2008;66(5): 1025-36

32. Díaz-Belenguer Á, Sánchez-Torres A, GayEscoda C. Role of Carnoy's solution in the treatment of keratocystic odontogenic tumor: A systematic review. Med Oral Patol Oral Cir Bucal. 2016;21(6):e689-95.

33. Costa Jr. AS, Miotto A, Paulo GA, Ferrari AP, Ota LH. Estudo experimental para avaliação de um sistema de baixo custo para crioterapia em spray. Einstein (São Paulo). 2019;17(2):1-5.

34. Cunha JF, Gomes CC, de Mesquita RA, Andrade Goulart EM, de Castro WH, Gomez RS. Clinicopathologic features associated with recurrence of the odontogenic keratocyst: a cohort retrospective analysis. Oral Surg Oral Med Oral Pathol Oral Radiol. 2016;121(6): 629-35.

35. Warburton G, Shihabi A, Ord RA. Keratocystic Odontogenic Tumor (KCOT/OKC) - Clinical Guidelines for Resection. J Maxillofac Oral Surg. 2015;14(3):558-64.

36. Madras J, Lapointe H. Keratocystic odontogenic tumour: reclassification of the odontogenic keratocyst from cyst to tumour. Tex Dent J. 2008;125:446-54. 


\section{CONFLITO DE INTERESSES}

Os autores declaram não haver conflitos de interesse

\section{AUTOR PARA CORRESPONDÊNCIA}

\section{Ellen Cristina Gaetti Jardim}

Faculdade de Odontologia - Faodo/UFMS

Av. Costa e Silva, S/N - Bairro Universitário

79070-900 Campo Grande - MS, Brasil

E-mail: ellen.jardim@ufms.br 